\title{
The ASTRI Project in the Framework of the Cherenkov Telescope Array
}

\author{
Stefano Vercellone* \\ INAF/IASF-Palermo \\ E-mail: stefanodifc.inaf.it
}

\section{on behalf of the ASTRI Collaboration}

http://www.brera.inaf.it/astri/

\section{and of the CTA Consortium}

https://portal.cta-observatory.org/

\begin{abstract}
The Cherenkov Telescope Array (CTA) will be the next generation facility to investigate the very high-energy gamma-ray emission from a large variety of celestial sources. The full array, installed at two sites, one in the northern and one in the southern hemisphere, will start to operate at the beginning of the next decade. In the meantime, a few telescope prototypes have been developed and some pre-production CTA telescopes have been planned. Within this framework, the Italian National Institute for Astrophysics (INAF) is leading the ASTRI project, whose aim is two-fold. The ASTRI Collaboration has successfully developed and installed in Sicily a prototype of the CTA small-sized telescopes (SST), according to an innovative dual-mirror (2M) optical solution and equipped with a silicon-based photo-detector Cherenkov camera. Moreover, INAF is leading the development of one of the mini-arrays of pre-production CTA telescopes composed of at least nine dual-mirror telescopes and proposed to be installed at the CTA southern site in 2018. The ASTRI mini-array of SST pre-production CTA telescopes will be able to both verify some of the adopted innovative solutions, such as the wide field of view, and to investigate sources emitting at energies from a few $\mathrm{TeV}$ up to hundreds of $\mathrm{TeV}$. We discuss the preliminary results obtained by the ASTRI SST-2M prototype during its ongoing commissioning phase, the expected Monte Carlo performance of the ASTRI mini-array of SST pre-production CTA telescopes, and provide an overview of the scientific topics that can be addressed both, as a stand-alone mini-array and in synergy with other pre-production CTA telescopes.
\end{abstract}

Frontier Research in Astrophysics-II

23-28 May 2016

Mondello (Palermo), Italy

\footnotetext{
* Speaker.
} 


\section{Introduction}

The very high-energy (VHE) portion of the electromagnetic spectrum (above $\approx 100 \mathrm{GeV}$ ) is currently being investigated by means of ground-based imaging atmospheric Cherenkov telescopes (IACTs, see [1] for a recent review). In order to dramatically boost the current IACT performance and to widen the VHE science, a new Cherenkov telescope array (CTA) has been proposed, as described in [2] and more recently in [3]. The wide energy range covered by the CTA (from a $20 \mathrm{GeV}$ up to $300 \mathrm{TeV}$ ) requires different classes of telescopes. The large-sized telescopes (LSTs, $\mathrm{D} \sim 23 \mathrm{~m}$ ) will lower the energy threshold down to a few tens of $\mathrm{GeV}$, the medium-sized telescopes (MSTs, D 12 m, SCTs, D 9.5 m) will improve by a factor of ten the sensitivity in the $0.1-10 \mathrm{TeV}$ energy range, and the small-sized telescopes (SSTs, primary mirror D $\sim 4 \mathrm{~m}$ ) will enhance Galactic plane investigations in the energy range beyond $100 \mathrm{TeV}$. To allow all-sky coverage, the full array will be installed at two sites, one for each hemisphere. About twenty telescopes (LST and MST) will be installed at both sites, covering an area of $\sim 1 \mathrm{~km}^{2}$, with LSTs at the center. The CTA southern site, covering an area of about $4 \mathrm{~km}^{2}$, will be completed with 70 SSTs. A detailed review of the CTA project is given in [4].

\section{The ASTRI Project and the ASTRI SST-2M Prototype}

Within the CTA framework, the Italian National Institute for Astrophysics (INAF) is leading the "Astrofisica con Specchi a Tecnologia Replicante Italiana" (ASTRI) Project [5]. Primarily,

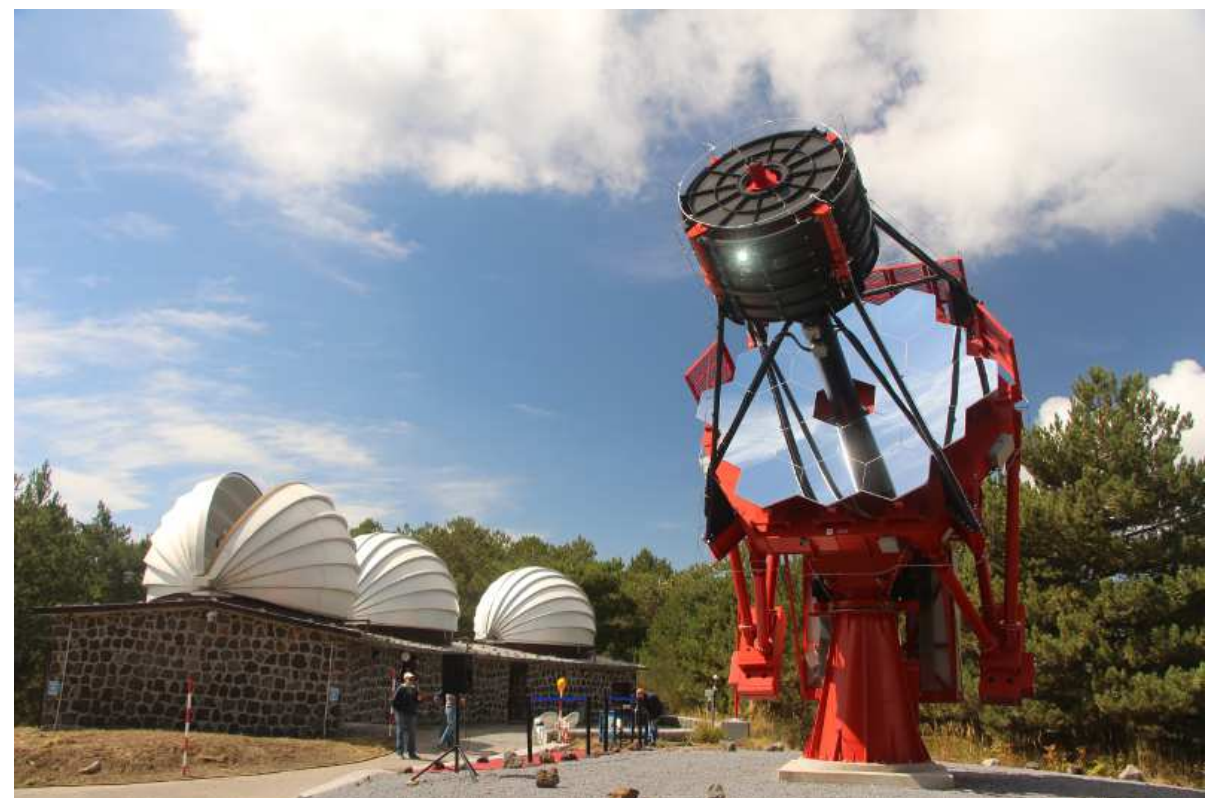

Figure 1: The ASTRI SST-2M prototype at the Serra La Nave observing station.

INAF has designed and developed an end-to-end prototype of the CTA small-sized telescope in a dual-mirror configuration (SST-2M). This prototype, inaugurated in September 2014, is currently being tested under field conditions at the INAF/OA-Catania "M.C. Fracastoro" observing station in Serra La Nave (Mount Etna, Sicily). Fig. 1 shows the prototype in front of the domes. Currently all 
telescope sub-systems are in place and properly working. The camera is completing its assembly, and it will be installed at the telescope focal plane in Fall 2016.

A dual-mirror optical design first proposed by Schwarzschild and Couder (SC) in 1926 has been recently re-proposed by [6] for the application in Cherenkov astronomy. It allows a better correction of aberrations at large incident angles even for small focal ratios and hence helps the construction of compact telescopes. In the SC telescope, the focal plane is located between two aspherical mirrors, close to the secondary mirror. No Cherenkov telescope has adopted this optical system before. The ASTRI SST-2M prototype acquired the first optical light of the star Polaris from a SC telescope in May $2015^{1}$.

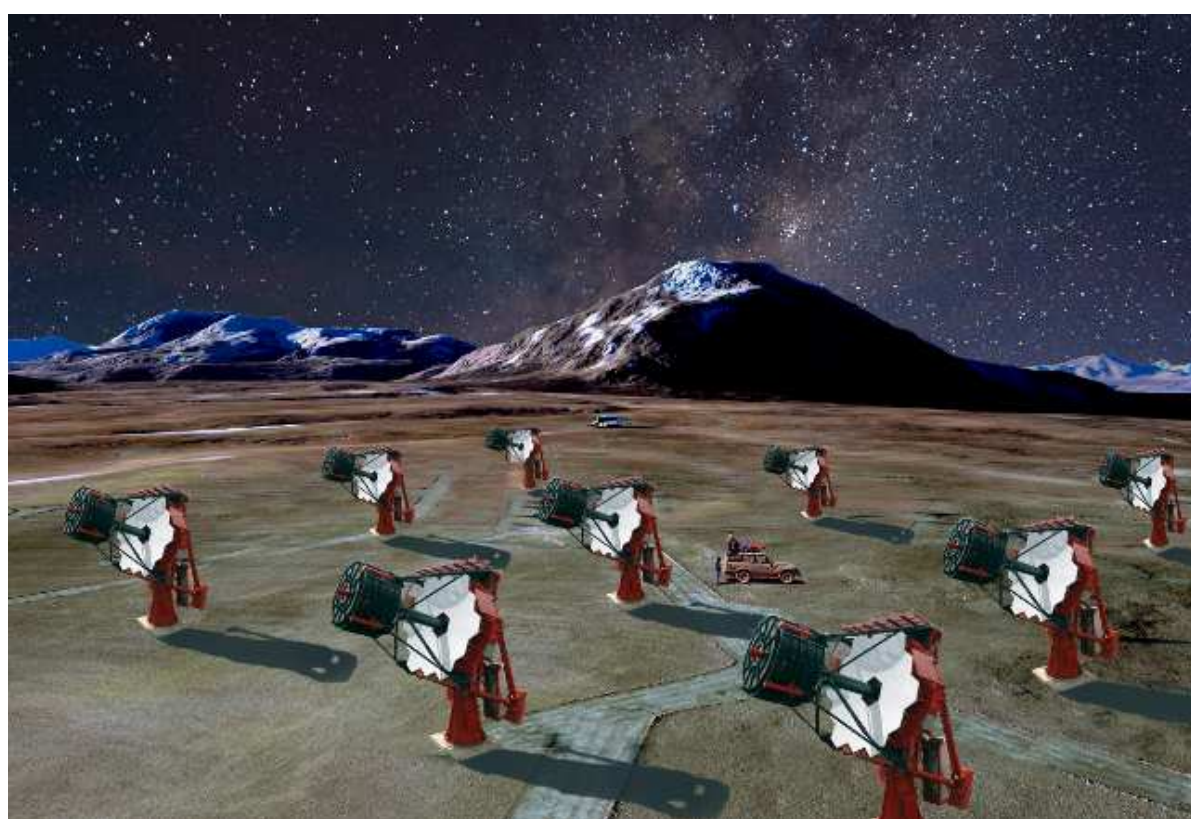

Figure 2: Artistic concept (not to scale) of the ASTRI mini-array of nine pre-production CTA small-sized telescopes.

The dual-mirror optical system will reduce the dimension, the weight, and the cost of the camera at the focal plane of the telescope, and will allow a more compact and stiffer mechanical structure, and an optimal imaging resolution across a wide field of view. Moreover, due to the reduced plate-scale, silicon-based photo-multipliers ( $\mathrm{SiPMs}$ ) can be adopted as light detectors.

The ASTRI SST-2M prototype adopts a segmented $4.3 \mathrm{~m}$ primary mirror (M1) composed of 18 facets, a monolithic $1.8 \mathrm{~m}$ secondary mirror (M2, with a radius of curvature of $2.2 \mathrm{~m}$ ), a focal length $\mathrm{F}=2.15 \mathrm{~m}$, a field of view $\mathrm{FoV} \sim 9.6^{\circ}$, for a ratio $\mathrm{F} / \mathrm{D}_{1}=0.5$. The mirror manufacturing process is the "glass cold shaping" technique, specifically developed by INAF for Cherenkov mirrors [7, 8]. The curved focal plane ( $\sim 1 \mathrm{~m}$ of radius of curvature) hosts 1984 logical pixels $(7 \mathrm{~mm} \times 7 \mathrm{~mm}$, or $\left.0.17^{\circ}\right)$. The current photo-sensors are Hamamatsu silicon-based photo-multipliers. The ASTRI camera [9] is extremely compact $(\sim 50 \mathrm{~cm} \times 50 \mathrm{~cm} \times 50 \mathrm{~cm})$ and light $(\sim 70 \mathrm{~kg})$. Contrary to other CTA telescopes adopting a signal-sampler front-end electronics (FEE), as FEE the ASTRI camera

\footnotetext{
${ }^{1}$ The image was obtained by means of a temporary CCD camera installed at the focal plane for mirror characterisation.
} 
adopts the CITIROC [10], a customized version of the EASIROC [11] ASIC signal-shaper manufactured by Omega ${ }^{2}$. Fig. 3 shows an ASTRI SST-2M camera image for an on-axis simulated

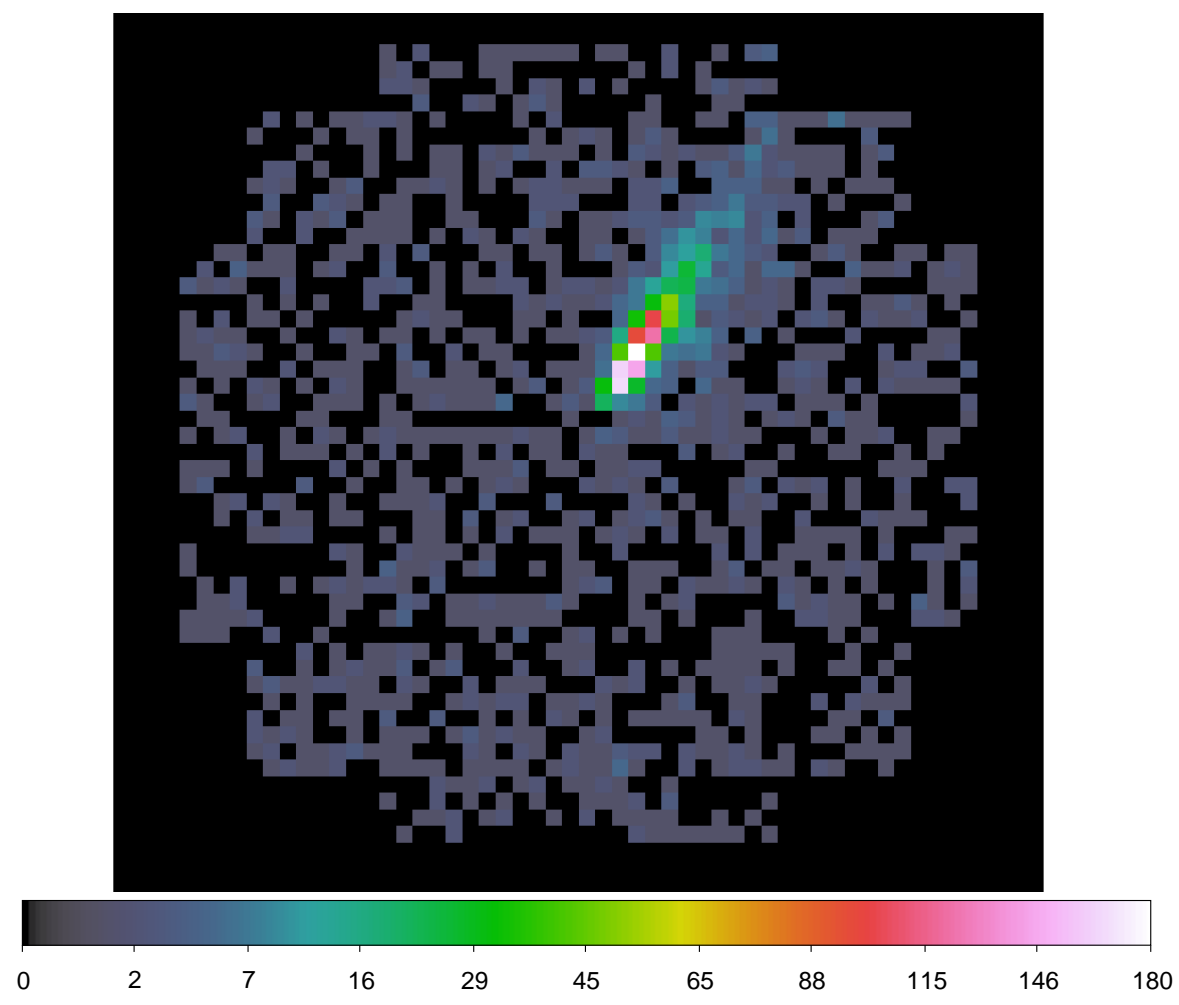

Figure 3: Image from the ASTRI SST-2M camera of an on-axis simulated gamma-ray event. The night-sky background is at a level of $1.9 \times 10^{12} \mathrm{ph} \mathrm{m}^{-2} \mathrm{~s}^{-1} \mathrm{sr}^{-1}$ (about three photo-electrons per pixel). The color-bar shows the number of photo-electrons per pixel.

primary gamma-ray event. The primary gamma-ray signal had an energy of $10 \mathrm{TeV}$ and a core distance of $142.77 \mathrm{~m}$.

We are completing the commissioning phase during which we tested all the mechanical and optical subsystems, as well as the control software. The commissioning of the camera will take place in October-November 2016. A science verification phase, during which we will observe the Crab Nebula, Mrk 421 and Mrk 501, will start immediately afterwards. The results of these observations will allow us to cross-check the prototype performance with our Monte Carlo simulations. A detailed description of the ASTRI SST-2M prototype data reconstruction and scientific analysis software is given in [12]. We estimate that, in the sensitivity range $(\mathrm{E} \geq 2 \mathrm{TeV})$, we can detect a flux level of $1 \mathrm{Crab}$ at $5 \sigma$ in a few hours [13]. Moreover, preliminary Monte Carlo simulations yield an energy and an angular resolution of about $20 \%$ and $0.1^{\circ}$ at $\mathrm{E}>1 \mathrm{TeV}$, respectively [14].

\section{The ASTRI Mini-array of CTA Pre-production Telescopes}

A remarkable improvement in terms of performance could come from the operation, during

\footnotetext{
${ }^{2}$ http: // omega.in2p3.fr/; manufactured under INAF intellectual property.
} 
2018, of a mini-array of pre-production CTA SSTs, composed of at least nine ASTRI telescopes (see Fig. 2, for an artistic concept) to be placed at the final CTA southern site.

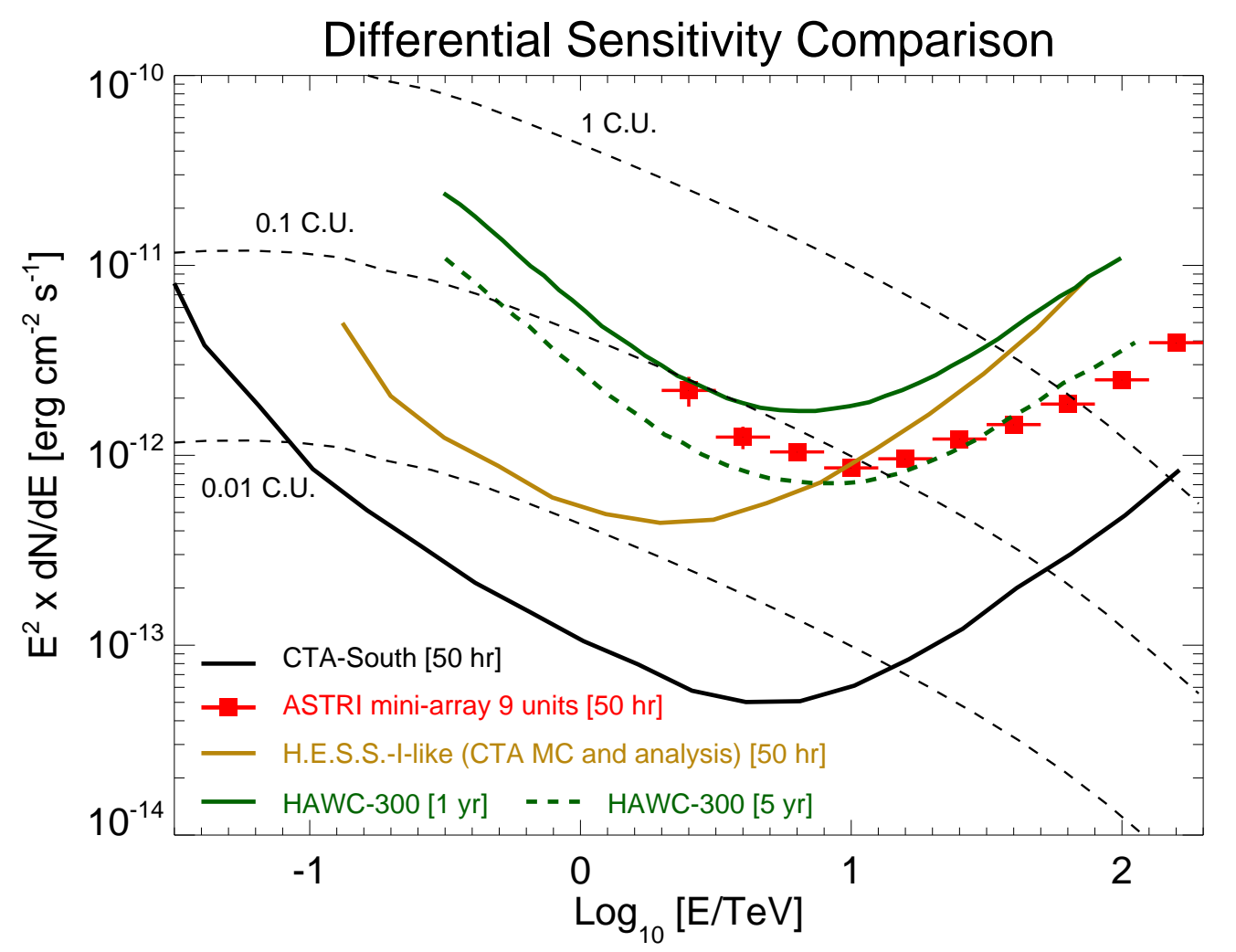

Figure 4: ASTRI mini-array of CTA pre-production SSTs differential sensitivity for 9 telescopes ( $50 \mathrm{hr}$, red points [15] compared to the CTA-South (50 hr, black curve [16]), H.E.S.S.-I-like (derived with CTA MC and analysis), (50 hr, golden curve [17]), and HAWC-300 (1 yr and $5 \mathrm{yr}$, solid and dashed green curves, respectively [18]) ones.

The ASTRI mini-array is a collaborative effort led by INAF in synergy with the University of São Paulo and FAPESP (Brazil) and the North-West University (South-Africa) and the Italian National Institute for Nuclear Physics (INFN). Preliminary Monte Carlo simulations [15] yield an improvement in sensitivity that, for nine telescopes, could surpass the H.E.S.S. sensitivity above $10 \mathrm{TeV}$, extending it up to about $100 \mathrm{TeV}$, as shown in Fig. 4. The ASTRI mini-array will be able to study in detail relatively bright (a few $\times 10^{-12} \mathrm{erg} \mathrm{cm}^{-2} \mathrm{~s}^{-1}$ at $10 \mathrm{TeV}$ ) sources with an angular resolution of a few arcmin and an energy resolution of about $10-15 \%$.

Fig. 4 also shows a comparison of the ASTRI mini-array differential sensitivity with respect to the HAWC-300 one (50 hr ASTRI, 1 and 5 yrs HAWC-300). Even if accessing different regions of the sky, HAWC-300 and the ASTRI mini-array will be able to monitor a few common sources and space regions, allowing us to investigate them in the same energy range (although with different sensitivities on short time-scales).

Thanks to the array approach, it will be possible to verify the wide FoV performance to detect very high energy showers with the core located at a distance up to $500 \mathrm{~m}$ and to compare the mini-array performance with the Monte Carlo expectations by means of deep observations of 


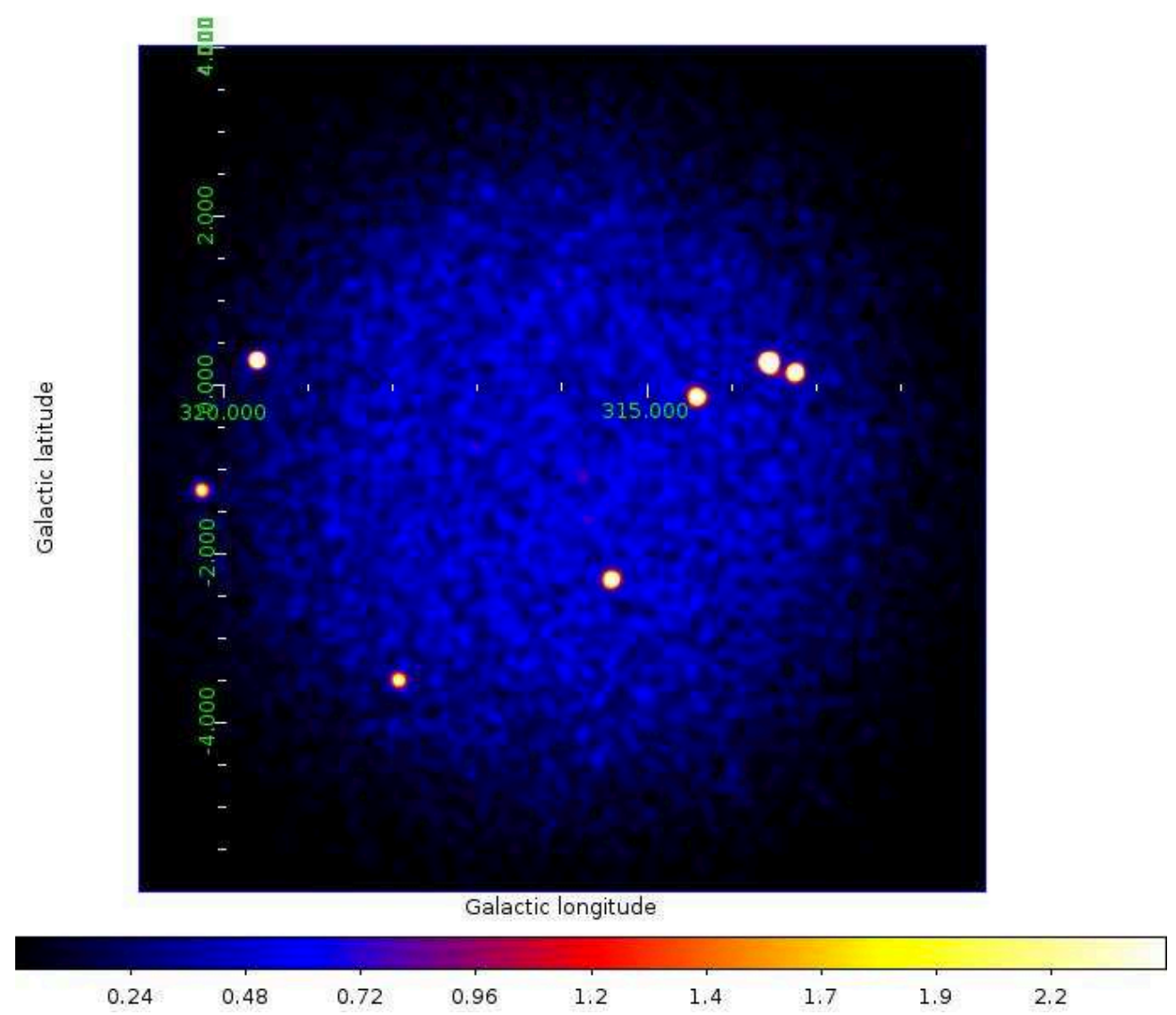

Figure 5: Simulation of a deep ASTRI mini-array observation (200 hr) of the Crux Galactic Arm.

a few selected targets. Moreover, it will be possible to perform the first CTA science, with its first solid detections during the first year of operation, as described in [19]. Prominent sources such as extreme blazars (KUV 00311-1938), nearby well-known BL Lac objects (Mrk 501) and radio-galaxies (M 87), galactic pulsar wind nebulae (Crab Nebula, Vela-X), supernovae remnants (Vela-junior, RX J1713.7-3946), as well as the Galactic Center can be observed in a previously unexplored energy range, in order to investigate the electron acceleration and cooling, relativistic and non relativistic shocks, the search for cosmic-ray (CR) PeVatrons, the study of the CR propagation, and the impact of the extragalactic background light on the spectra of the sources.

A relevant innovation with respect the current IACTs is the wide optical FoV of the CTA SSTs $\left(>9^{\circ}\right)$. Although the sensitivity will drop by a factor of two at $3^{\circ}$ off-axis angle, the chance to detect more than one source along the Galactic plane is quite high. Fig. 5 shows a simulation of a deep ASTRI mini-array observation $(200 \mathrm{hr})$ of the Crux Galactic Arm. The counts-map, in Galactic coordinates, has a pixel size of $0.02^{\circ}$. The color-bar shows the intensity in units of

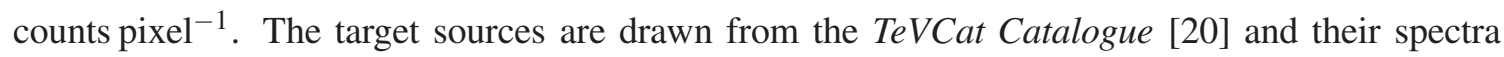
have been extrapolated above $\sim 10 \mathrm{TeV}$ according to literature.

Fig. 6 shows the SED of the extreme blazar 1ES 0229+200 with superimposed different theoretical SED fits (both hadronic and leptonic) assuming different EBL models (see [21] for a detailed 


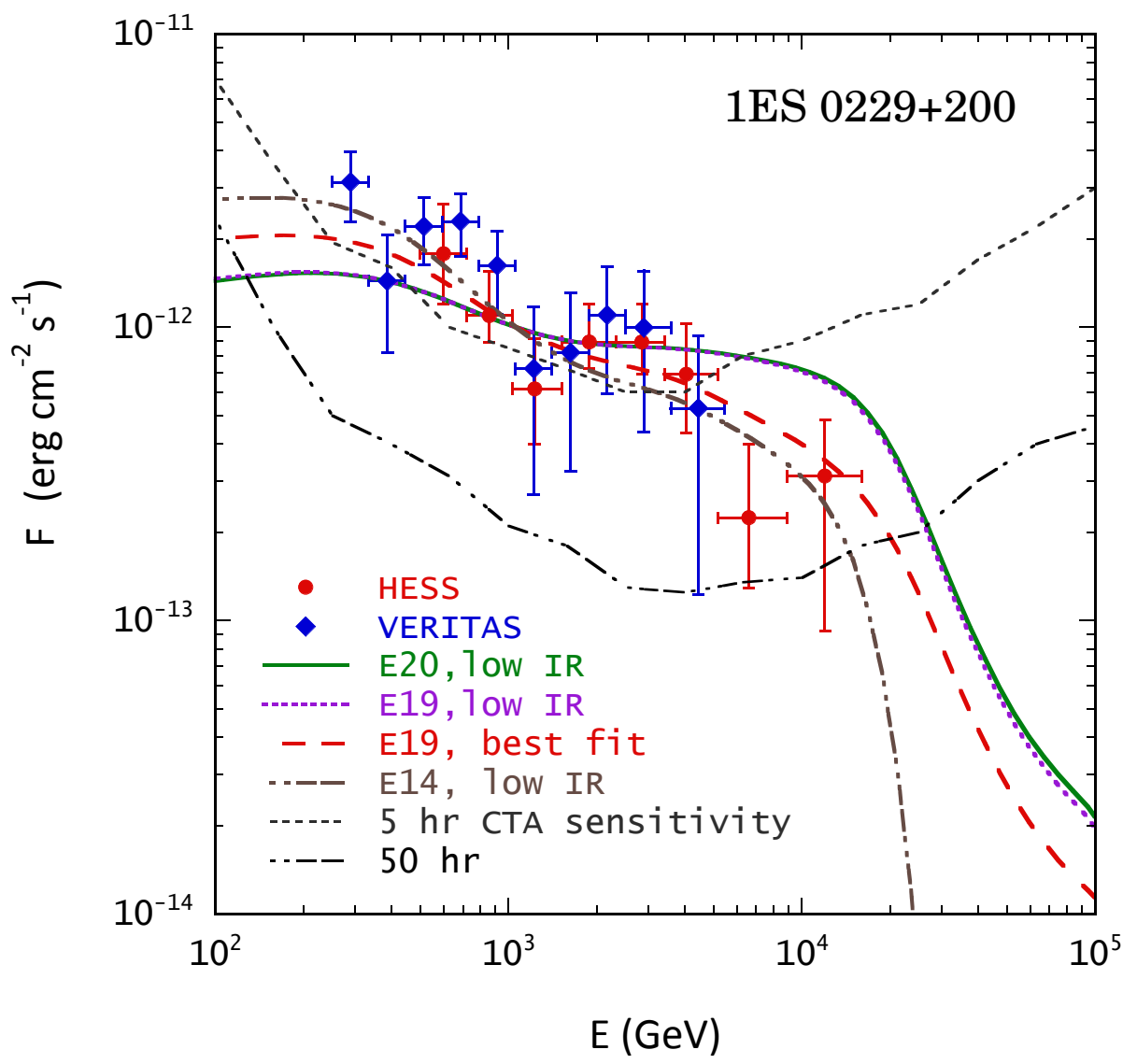

Figure 6: Spectral energy distribution of the extreme blazar 1ES 0229+200 (see [21] for details).

discussion) and the 5- $\sigma$ differential sensitivity for 5 and $50 \mathrm{hr}$ observations with CTA (configuration $\mathrm{E}$, as reported in [2]). Because of the uncertainty in EBL models, it is not easy to distinguish between the hadronic and leptonic scenarios at $\sim 1-10 \mathrm{TeV}$ energies. At higher energies, however, UHECR-induced cascade emission becomes harder than the gamma-ray one. A detection of $\geq 25 \mathrm{TeV}$ gamma-rays from 1ES $0229+200$ would only be consistent with an hadronic scenario.

\section{Conclusions}

The ASTRI mini-array of pre-production CTA SSTs will operate when the the current generation of IACTs will still be active. Compared to them, the ASTRI mini-array will extend the sensitivity up to $100 \mathrm{TeV}$ and beyond, a never-explored energy range by IACTs. Moreover, it will benefit from a much larger field of view which will allow us to study in detail extended sources at energies about a decade higher than what is currently being explored and to monitor simultaneously a few close-by sources during the same pointing. Long exposures will be preferred, restricting the number of possible targets, and extending the observations also during brighter Moon periods, thanks to the use of a SiPMs-based camera.

The lower imaging energy threshold of current and future extended air-shower (EAS) detectors $(\sim 100 \mathrm{GeV})$ and the wider energy range of the ASTRI mini-array (beyond $100 \mathrm{TeV})$ will allow us 
a direct comparison of scientific data (spectra, light-curves, integral fluxes) of those sources which could be monitored simultaneously, although on different integration time-scales. Moreover, the high-energy boundary of both EAS and the ASTRI mini-array will allow us to study the VHE $(\mathrm{E} \geq 10 \mathrm{TeV})$ emission from extended sources, such as Supernovae Remnants and Pulsar-Wind Nebulae, and to investigate the presence of spectral cut-offs. In summary, the ASTRI mini-array could be considered as one of the first CTA seeds, allowing the entire CTA Consortium to start seminal studies on both Galactic and extra-galactic sources.

\section{Acknowledgment}

This work is supported by the Italian Ministry of Education, University, and Research (MIUR) with funds specifically assigned to the Italian National Institute of Astrophysics (INAF) for the Cherenkov Telescope Array (CTA), and by the Italian Ministry of Economic Development (MISE) within the "Astronomia Industriale" program. We acknowledge support from the Brazilian Funding Agency FAPESP (Grant 2013/10559-5) and from the South African Department of Science and Technology through Funding Agreement 0227/2014 for the South African Gamma-Ray Astronomy Programme. We gratefully acknowledge support from the agencies and organizations listed under Funding Agencies at this website: http://www.cta-observatory.org/.

\section{References}

[1] J.A. Hinton, W. Hofmann, Teraelectronvolt Astronomy, Annual Review of Astronomy \& Astrophysics 47 (2009) 523 [arXiv: 1006.5210$]$

[2] M. Actis et al., Design concepts for the Cherenkov Telescope Array CTA: an advanced facility for ground-based high-energy gamma-ray astronomy, Experimental Astronomy 32 (2011) 193 [arXiv:1008.3703]

[3] B.S. Acharya et al., Introducing the CTA concept, Astroparticle Physics 43 (2013) 3

[4] J. Carr, The new gamma-ray observatory: CTA, EPJ Web of Conferences 121 (2016) 04004

[5] G. Pareschi for the ASTRI Collaboration, the GATE Collaboration, the CHEC Collaboration, the CTA Consortium, The dual-mirror Small Size Telescope for the Cherenkov Telescope Array, Proceedings of the $33^{\text {rd }}$ ICRC (2013) [arXiv: 1307 . 4962]

[6] V. Vassiliev, S. Fegan, P. Brousseau, Wide field aplanatic two-mirror telescopes for ground-based $\gamma$-ray astronomy, Astroparticle Physics 28 (2007) 10 [astro-ph / 0612718 ]

[7] R. Canestrari et al., The ASTRI SST-2M prototype for the CTA: manufacturing of the structure and the mirrors, SPIE Conference Series 9145 (2014) 91450M

[8] R. Canestrari et al., The glass cold-shaping technology for the mirrors of the CTA, SPIE Conference Series 9151 (2014) 91512V

[9] O. Catalano et al., The camera of the ASTRI SST-2M prototype for the Cherenkov Telescope Array, SPIE Conference Series 9147 (2014) 91470D

[10] D. Impiombato et al., Characterization and performance of the ASIC (CITIROC) front-end of the ASTRI camera, Nuclear Instruments and Methods in Physics Research A 794 (2015) 185

[arXiv: 1506.00264$]$ 
[11] S. Callier et al., EASIROC, an Easy \& Versatile ReadOut Device for SiPM, Physics Procedia 37 (2012) 1569 (2012)

[12] S. Lombardi et al., ASTRI SST-2M prototype and mini-array data reconstruction and scientific analysis software in the framework of the Cherenkov Telescope Array, SPIE Conference Series in press (2016)

[13] C. Bigongiari, the ASTRI Collaboration, Expected performance of the ASTRI-SST-2M telescope prototype, Proceedings of the $33^{\text {rd }}$ ICRC (2013) [arXiv: 1307.5006 ]

[14] C. Bigongiari, the ASTRI Collaboration, the CTA Consortium, Simulation of the ASTRI two-mirrors small-size telescope prototype for the Cherenkov Telescope Array, Journal of Physics Conference Series 718 (2016) 052003

[15] F. Di Pierro, the ASTRI Collaboration, the CTA Consortium, Expected performance of the ASTRI mini-array in the framework of the Cherenkov Telescope Array, Journal of Physics Conference Series 718 (2016) 052008

[16] The CTA Consortium, CTA Performance, https://portal.cta-observatory.org/Pages/CTA-Performance.aspx

[17] K. Bernlöhr, Private communication

[18] A. U. Abeysekara et al., Sensitivity of the high altitude water Cherenkov detector to sources of multi-TeV gamma rays, Astroparticle Physics 50 (2013) 26 [arXiv:1306.5800]

[19] S. Vercellone, the ASTRI Collaboration, The ASTRI Mini-Array Science Case, Proceedings of the $33^{\text {rd }}$ ICRC (2013) [arXiv:1307.5671]

[20] S. Wakely, D. Horan, TeVCat, http://tevcat2.uchicago.edu/

[21] K. Murase et al., Blazars as Ultra-high-energy Cosmic-ray Sources: Implications for TeV Gamma-Ray Observations, Astrophysical Journal 749201263 [arXiv: 1107.5576 ] 